\title{
Persistence of Ion Cyclotron Waves and Stochasticity of Kinetic Alfvén Waves in the Solar Wind
}

\author{
Daniele Telloni
}

check for

updates

Citation: Telloni, D. Persistence of Ion Cyclotron Waves and Stochasticity of Kinetic Alfvén Waves in the Solar Wind. Atmosphere 2021, 12, 44. https://doi.org/10.3390/atmos 12010044

Received: 11 November 2020 Accepted: 28 December 2020 Published: 30 December 2020

Publisher's Note: MDPI stays neutral with regard to jurisdictional clai$\mathrm{ms}$ in published maps and institutional affiliations.

Copyright: () 2020 by the author. Licensee MDPI, Basel, Switzerland. This article is an open access article distributed under the terms and conditions of the Creative Commons Attribution (CC BY) license (https:// creativecommons.org/licenses/by/ $4.0 /)$.
National Institute for Astrophysics, Astrophysical Observatory of Torino, Via Osservatorio 20, 10025 Pino Torinese, Italy; daniele.telloni@inaf.it; Tel.: +39-011-8101-984

\begin{abstract}
This paper investigates the nature of the physical processes underlying the origin of the Ion Cyclotron Waves (ICWs) and Kinetic Alfvén Waves (KAWs) in the solar wind, by studying their Waiting Time Distributions (WTDs). The results show that ICWs and KAWs do not share common statistical properties: while KAWs independently occur as stochastic, uncorrelated wave packets governed by Poisson statistics, ICWs are highly correlated, thus departing from the Poisson hypothesis. The results based on the WTD analysis may cast more light on the mechanisms actively at work in the generation of the two wave modes. Specifically, while the stochastic character of KAWs may be reminiscent of the random convection-driven jostling of the flux-tube foot-points that generates the Alfvén waves in the lower solar atmosphere, the correlations among the ICW events can be effectively explained on the basis of the persistent nature of the mechanism underlying the local origin of ICWs, namely the proton cyclotron instability. Alternative explanations for the observed distribution of ICW waiting times, based on a piecewise-constant Poisson process involving time-varying rates, are also reported.
\end{abstract}

Keywords: magnetohydrodynamics; space plasmas; solar wind; interplanetary turbulence; alfvén waves

\section{Introduction}

Over the last 60 years or so a fleet of space missions dedicated to the exploration of the Sun and the interplanetary space has led to countless and startling discoveries, advancing our knowledge of the Sun's activity and of its influence in the whole solar system, through the solar wind, a continuous flow of magnetized plasma emitted by the outer layer of the solar atmosphere (the solar corona), and inflating a bubble-like cavity of space known as heliosphere, which is bounded from the interstellar medium by the heliopause, where the solar wind pressure is balanced by that of stellar winds of the surrounding stars. Achievements in solar and space physics have shed light on fundamental plasma physical processes ubiquitous in the Universe, such as turbulence and magnetic reconnection, that are relevant to a much broader astrophysical community and thus impacting also on other physics research fields.

The event that opened a new era in the investigation of the interplanetary space was the first ever direct in-situ measurement, in 1962 by Mariner 2 on its way to Venus, of the solar wind [1], which had been theoretically predicted in the mid-1950s by Parker [2]. Since then, a large number of observations either confirmed what theory had anticipated, such as the Parker spiral configuration of the interplanetary magnetic field [2] and the highly turbulent nature of the solar wind [3], or led to new discoveries, such as large eruptions of magnetized plasma from the solar corona into the heliosphere, known as Coronal Mass Ejections CMEs [4]. Overall, more than 50 years of study of the interplanetary space and the solar wind-related phenomena led to a rather complete mapping and characterization of the large-scale structures permeating the whole heliosphere. Specifically, it turns out that the solar wind is highly structured and varies both in space and in time over the course of the solar cycle. 
During low solar activity phases, the solar wind has been observed, by the early 1970s, to occur in two distinct states, namely as fast and slow solar wind, though their differences extend well beyond their speeds e.g., [5]. While high-speed streams primarily come from persistent polar coronal holes (high-latitude rarefied regions of open magnetic field) and thus fill mainly the high heliographic latitudes ([6], though low-latitudinal extensions can reach the ecliptic plane), the origin of the near-ecliptic slow solar wind is still largely debated. Due to the 27-day solar rotation, the solar wind becomes a complex wavy spiral of alternating high- and low-speed flows, which forms and shapes the largest structure in the solar system, the Heliospheric Current Sheet (HCS). At the interfaces between high-speed streams overtaking the slower solar wind ahead, Co-Rotating Interaction Regions (CIRs), namely regions of enhanced density and magnetic field strength, form. The reader is referred to the excellent book by Hundhausen [7] for a complete overview of the solar wind and heliosphere based on early space-based observations of the interplanetary medium. A more recent review of the observations of the solar corona from space is provided by Antonucci et al. [8].

As Sun's activity increases, the solar corona dramatically reconfigures: polar coronal holes shrink until they disappear completely (while smaller coronal holes emerge at lower latitudes), and the solar surface fills with a large number of active regions and other complex structures. As a result, the slow solar wind, no more spatially limited to just the HCS, becomes the dominant flow state, filling the heliosphere at high latitudes too [9]. During the highest Sun's activity phase, the solar wind is also largely perturbed by CMEs, which occur up to an order of magnitude more frequently at solar maximum than at minimum e.g., [10]. Such transients can reach such high velocities to accelerate Solar Energetic Particles SEPs, [11].

Since the first ever evidence for highly correlated plasma velocity and magnetic field fluctuations in the solar wind by Belcher and Davis [12] in 1971 using Mariner 5 data, which marks de facto the first observation of Alfvén waves in the inner heliosphere, the Alfvénic and turbulent properties of the solar wind have been extensively studied in-situ by a series of interplanetary probes orbiting at different distances from the Sun (as the pioneering Helios missions, which in the 1970s approached the Sun at $0.3 \mathrm{AU}$, and the Voyager missions, the most distant deep-space probes, which both reach the final frontier of the heliosphere, entering the interstellar medium, more than 30 years after their launch) as well as in and out of the ecliptic (as Ulysses which, from its polar orbit, provided the first in-situ measurement of the high-latitude heliosphere).

The picture depicted is that the solar wind is a highly turbulent medium (as suggested by the ubiquitous observation of Kolmogorov-like scaling governing the amplitude of the fluctuations at inertial scales) that exhibits a self-similar nature: magnetic field and velocity fluctuations show the same statistical properties regardless the timescale considered. This self-similarity (or scale invariance) holds in the scale range from the correlation length down to the proton gyroradius [13]. For decades the greatest effort has been mainly made in the study of how energy is transferred from large to small scales in the turbulent decay, in the investigation of the evolution of the turbulent spectrum as the wind expands, and in the characterization of the fast and slow solar wind in terms of their turbulent properties (such as Alfvénicity, magnetic compressibility, intermittency). This has been leading to view the solar wind as a natural turbulence laboratory (see the extensive and comprehensive review by [14], and references therein).

Due to instrumentation limitations imposed by the relatively low-cadence measurement acquisition, which prevent the exploration of high-frequency fluctuations, the investigation of the scales where turbulently-transferred energy is eventually dissipated to heat the solar wind plasma and of the related processes has been poorly dealt with from an observational perspective [13]. Only recently, the high-frequency regime of solar wind fluctuations has been increasingly studied, unraveling the existence of two different populations, with opposite polarization states and differently orientated wavevectors, at scales typical of proton kinetics. Specifically, right- and left-handed magnetic fluctuations, 
with wavevectors respectively transverse (or highly oblique) and nearly parallel to the local magnetic field, have been clearly resolved only about ten years ago $[15,16]$ at scales around proton characteristic lengths (as the proton inertial length, the proton Larmor radius or the proton gyroradius, $\Omega_{p}$ ) and attributed to Kinetic Alfvén Waves (KAWs) and outwardly propagating Ion Cyclotron Waves (ICWs), respectively.

Since then, the nature of the two different families of fluctuations has been deeply investigated. In particular, KAWs have been found fo have a larger amplitude and to be more compressive with respect to ICWs [17,18]. Later, Telloni and Bruno [19] showed, for the first time, that ICWs and KAWs lie in distinct regions of the plasma instabilitytemperature anisotropy $\beta_{\|}-T_{\perp} / T_{\|}$plane: as a matter of fact, KAWs are mainly characterized by $\beta_{\|}$values close to 1 and a nearly temperature isotropy, while ICWs exhibit a $\beta_{\|}$lower than 1 and a temperature anisotropy generally higher than 1 . By exploiting a statistical approach, Telloni et al. [20] have shown compelling evidences for a clear link of the amplitude of the Alfvénic fluctuations and the proton temperature anisotropy at fluid scales, with the presence of ICWs at kinetic scales, thus providing robust hints in favor of a scenario in which the proton cyclotron instability might be the likely mechanism for the generation of ICWs in the solar wind. According to this interpretation, high-frequency Alfvén waves would resonantly interact with protons around $\Omega_{p}$, increasing their thermal energy perpendicularly to the magnetic field and, in turn, their temperature anisotropy. As a result, the proton Velocity Distribution Functions (VDFs) would depart from thermal equilibrium, triggering the proton cyclotron instability to restore the VDFs towards an isotropic condition, through the release of the excess of energy built up in the anisotropic VDFs via the generation of ICWs.

Despite the considerable advances made during the last decade in the study of the nature of the solar wind fluctuations in the dissipation range, many key scientific questions remain still unanswered, especially those related to the origin of wave modes at proton scales and their role in plasma heating with respect to the actual mechanisms at work. The advent of Parker Solar Probe and Solar Orbiter with their unprecedentedly high temporal resolution in both magnetic and plasma measurements, combined with comprehensive theory and modeling efforts, will represent an effective response to these challenges. Thanks to Parker Solar Probe and Solar Orbiter, the next decade of research can be expected to lead to advances in the understanding of fundamental physical processes occurring in space plasmas, providing new physical insights about the nature of turbulence in space plasma and, more importantly, about the coupling between kinetic turbulence and magnetohydrodynamic (or fluid) turbulence, which represents the fascinating next frontier of exploration in plasma physics.

Not so far addressed in the study of plasma waves in solar wind turbulence, this paper reports the study of the statistical properties of ICWs and KAWs, namely of the distribution of the time interval between two successive wave packets. Since the analysis of the Waiting Time Distributions (WTDs) of ICWs and KAWs provides information regarding whether these wave modes occur as independent or correlated events, it is useful in casting some more light on the nature of the processes underlying their generation/dissipation, on their similarities and differences, and on their relationship with the mechanisms responsabile for the conversion of magnetic energy into particle thermal and kinetic energy. This is the aim of the present paper, which is organized as follows: Section 2 presents the analysis results, while discussion on the physical relevance of ICW and KAW packet time distribution in the light of the mechanisms driving their origin, and concluding remarks are reported in Section 3.

\section{Data Analysis}

The analysis refers to the same time interval from 00:00 to 13:24 on 1 July 2010 already studied in previous works by the same author [17-19], when the Magnetic Field Investigation MFI, [21] instrument on board the Wind spacecraft sampled the trailing edge of a high-speed stream, characterized by large amplitude Alfvénic fluctuations. Following 
Telloni and Bruno [19], packets of ICWs and KAWs have been extracted from the angle distribution of the normalized magnetic helicity $\sigma_{m}$ (not shown here, refer to the right panel of their Figure 1) as trains of left- and right-handed fluctuations singled out at the frequency of $0.7 \mathrm{~Hz}$ (characteristic of both the wave modes) and at quasi-parallel and quasi-perpendicular sampling angles with respect to the local background magnetic field $\mathbf{B}_{0}$. Specifically, the criteria for magnetic fluctuations to be selected as ICWs or KAWs are based on the following requirements: (i) the normalized magnetic helicity is larger (smaller) than +0.5 (-0.5) for ICWs (KAWs); (ii) the angle between the solar wind velocity and the local mean magnetic field is in the range $140^{\circ}-180^{\circ}\left(70^{\circ}-110^{\circ}\right)$ for ICWs (KAWs); (iii) the frequency of $0.7 \mathrm{~Hz}$ (which is to be considered just as a reference frequency rather than the monochromatic frequency of the wave) is arbitrarily chosen to sample ICWs and KAWs, since this crosses both the wave population cores in the $\sigma_{m}$ angle distribution (see right panel of Figure 1 in [19]). These conditions ensure that the selected magnetic field data points belong to left-(right-)handed polarized fluctuations at sub-ion scales, propagating at quasi-parallel(-perpendicular) angles with respect to $\mathbf{B}_{0}$, and thus interpreted as ICWs (KAWs). Since the sign of $\sigma_{m}$ depends on the orientation of $\mathbf{B}_{0}$, these considerations apply to inward magnetic sectors (as in the present paper), while opposite values of $\sigma_{m}$ have to be considered for outward magnetic sectors, where ICWs propagate at angles smaller than $40^{\circ}$ with respect to $\mathbf{B}_{0}$ see [15]. Then, trains (say packets) of ICWs (KAWs) are identified as consecutive ICWs (KAWs) events: the start and end of each wave packet are thus determined as the first and last of successive events. On average, each wave packet lasts about $1 \mathrm{~s}$, being made up of a dozen data points at $92 \mathrm{~ms}$ resolution. This implies that the duration of the wave packets is less than the average waiting time between two consecutive events, ensuring that the process is point-like, which is necessary to perform the statistical analysis based on waiting times. $523 \mathrm{ICW}$ and $180 \mathrm{KAW}$ packets are thus found in the selected time period.

However, caution must be taken considering that the identification is based solely on the magnetic helicity. Indeed, while from linear theory, ICWs are expected to be left-handed polarized, for KAWs the polarization signature is less clear. As a matter of fact, in the wavenumber range corresponding to proton gyroscale, the kinetic Alfvén wave branch has a polarization which is more linear rather than right-handed. In addition, KAWs extend in a wider wavenumber range with respect to ICWs, as well as at angles not only perpendicular, but also oblique, to $\mathbf{B}_{0}$. This might lead to potentially missing some wave packets corresponding to KAWs, thus underestimating their number. Finally, since ICWs propagate along $\mathbf{B}_{0}$, while KAWs advect perpendicular to $\mathbf{B}_{0}$, their detection depends on the spacecraft sampling direction: moving along $\mathbf{B}_{0}$ helps to capture ICWs rather than KAWs (though actually present). However, the existence of ICWs and KAWs is not regulated by the sampling direction, rather by physical quantities such as the parallel proton plasma beta and temperature anisotropy, as clearly shown in $[19,20]$. While a detailed and critical analysis on how the selection criteria based exclusively on magnetic helicity affects the ICW and KAW statistics has yet to be performed (addressing, for instance, how the ICW and KAW identification depends on the assumed $\sigma_{m}$ threshold, as well as on the wavenumber and angle ranges considered during the selection), it is worth reminding that this selection method is the same successfully adopted in Telloni and Bruno [19], which led to the first observational evidence that ICWs and KAWs are characterized, as expected, by different temperature anisotropies and parallel plasma beta values and, in turn, governed by different plasma instability/processes.

The waiting times $\Delta t$ between two successive KAW and ICW packets are displayed respectively in the top-left and bottom-left panel of Figure 1, as a function of the event occurrence time. The corresponding WTDs $P(\Delta t)$ sampled in intervals of $1 \mathrm{~s}$ are shown, for $\Delta t \leq 30 \mathrm{~s}$, in the right-side panels of the same figure. The blue curves represent the theoretical distributions expected under Poisson statistics for both ICW and KAW packets: these are given by $P(\Delta t)=\bar{\lambda} e^{-\bar{\lambda} \Delta t}$, where $\bar{\lambda}=0.112 \mathrm{~s}^{-1}$ and $=0.325 \mathrm{~s}^{-1}$ are the average KAW 
and ICW packet occurrence rates, estimated within a time window of $30 \mathrm{~s}$ spanning the analyzed time interval.

Time evolution of KAWs waiting times

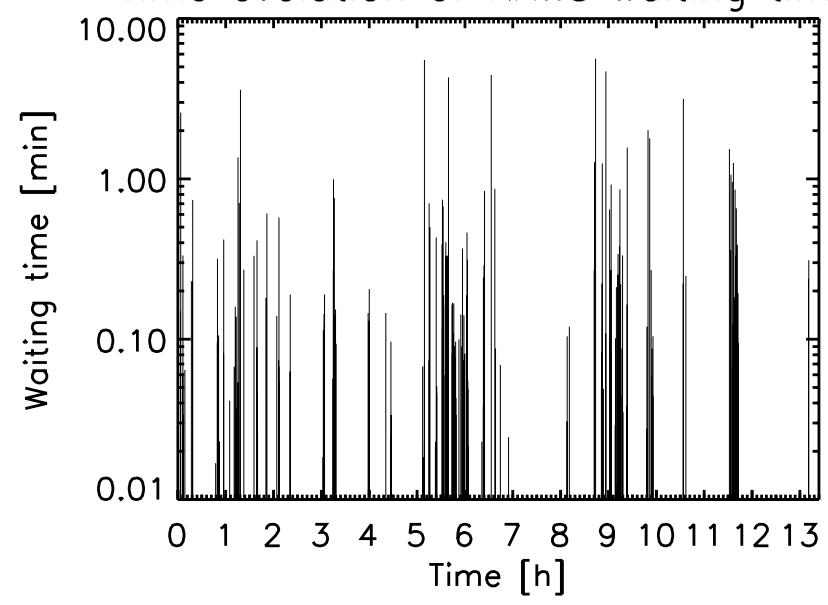

Time evolution of ICWs waiting times

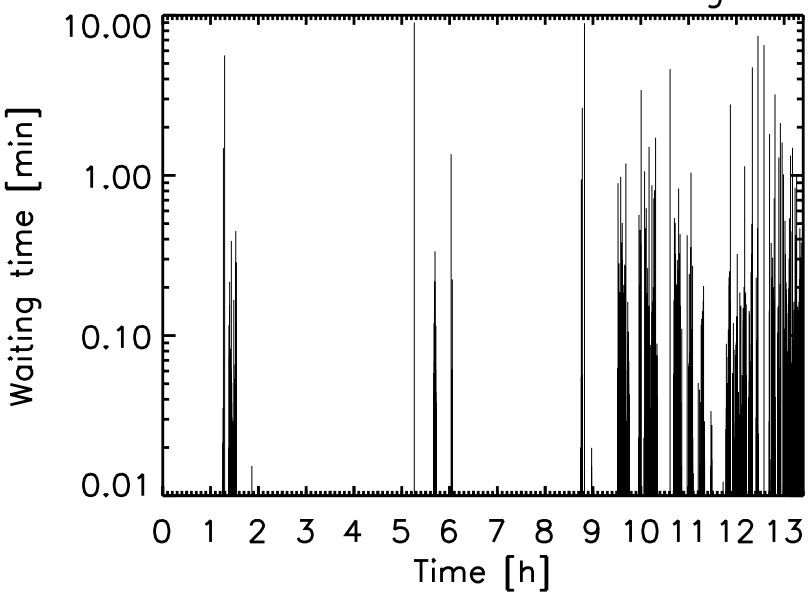

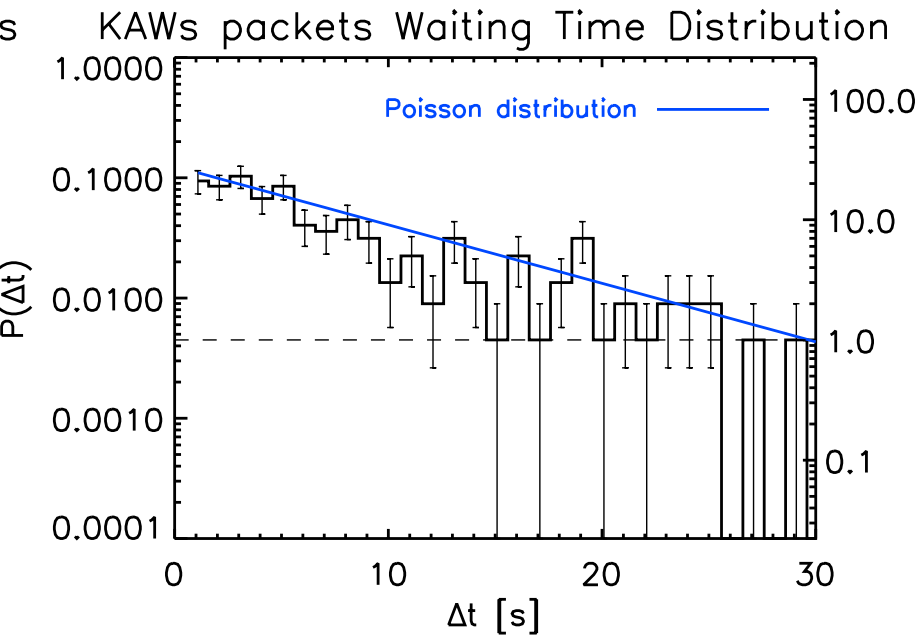

ICWs packets Waiting Time Distribution

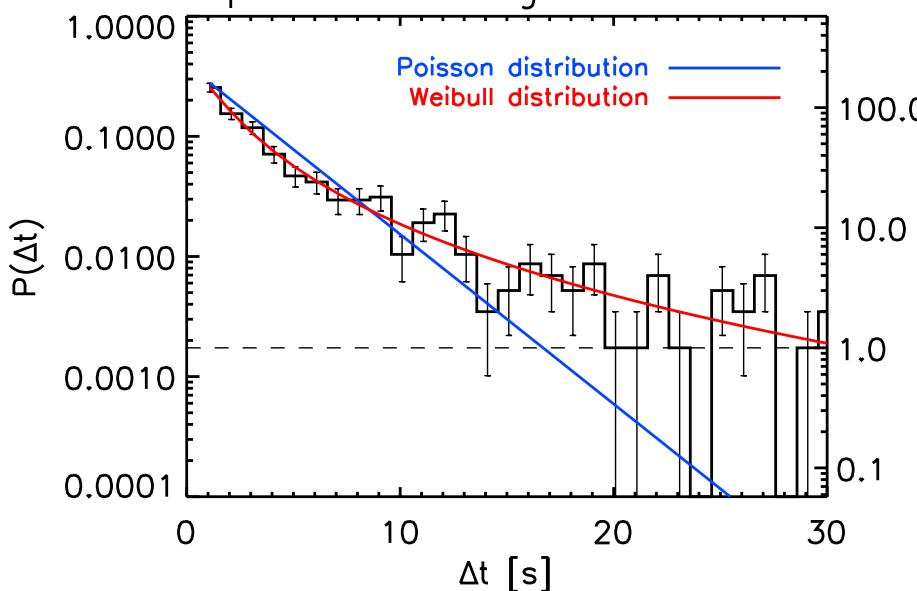

Figure 1. Sequence of the waiting times $\Delta t$ at the event occurrence time (left panels) and corresponding WTDs $P(\Delta t)$ sampled in 1-second bins (right panels) for KAW (top panels) and ICW (bottom panels) packets; the corresponding theoretical Poisson distributions are displayed as blue curves; the ICW WTD is fitted by an exponentiated Weibull function (red curve), representing a persistent process with some memory generating correlations among the waiting times; the horizontal dashed lines refer to a frequency of occurrence equal to 1.

A first glance clearly indicates that while the KAW WTD can be well reproduced by the Poisson function within the uncertainties, the ICW $P(\Delta t)$ is inconsistent with Poisson statistics. Indeed, the ICW WTD is remarkably lower than the Poisson distribution for $\Delta t \lesssim 8 \mathrm{~s}$, indicating that ICW packets are clustered at scales smaller than a few seconds. Conversely, $P(\Delta t \gtrsim 8)$ is higher than Poisson expectations, suggesting that ICW trains occur less frequently than if their manifestation were governed by a stochastic process and, in turn, that voids are present in the ICW time series at scales larger than a few seconds. As a matter of fact, for long waiting times, a power-law tail $\propto \Delta t^{-\gamma}$ with scaling index $\gamma=2.46 \pm 0.02$ is exhibited by the ICW distribution. It appears thus evident that while KAW packets occur as random (i.e., uncorrelated) events, observations are inconsistent with a stochastic occurrence of ICW: rather, long-term correlations are present among ICW packets, which manifest as short clusters separated by relatively long quiet intervals consistent with previous works that suggest that ICWs occur in 'storms' [22,23].

A closer analysis of the ICW WTD, attempting to better characterize their temporal clustering or voiding, leads to the observed distribution of waiting times being fitted with an exponentiated Weibull function (red curve in the bottom-right panel of Figure 1), which 
accounts for the presence of correlations among the waiting times. It arises by simply assuming that the events in the system are correlated to some degree. The WTD of any class of events can be indeed expressed as

$$
P(\Delta t)=z(\Delta t) e^{-\int_{0}^{\Delta t} z(x) d x},
$$

where $z(\Delta t)$ represents the local probability that an event will occur after that a time interval $\Delta t$ has elapsed since the previous one. In a stochastic (i.e., memoryless) process this probability is constant with time, $z(\Delta t)=\lambda$, thus retrieving the classical Poisson distribution $P(\Delta t)=\lambda e^{-\lambda \Delta t}$. By contrast, if a certain amount of memory is present in the system, then the occurrence probability varies with time. Hence, $z(\Delta t)$ can be expressed as

$$
z(\Delta t)=\lambda^{k} k \Delta t^{k-1},
$$

where the parameter $k$ describes the statistical properties of the events: if $k \lessgtr 1$, then the occurrence probability decreases (increases) with the increasing time interval between two successive events, thus implying the presence of clusters (voids) in the system. It is worth noting that $k=1$ trivially reduces to $z(\Delta t)=\lambda$ and, in turn, to the Poisson distribution function. According to Equation (2), Equation (1) becomes the Weibull probability distribution function [24]

$$
P(\Delta t)=\frac{k}{\beta}\left(\frac{\Delta t}{\beta}\right)^{k-1} e^{-\left(\frac{\Delta t}{\beta}\right)^{k}}
$$

where $\beta=1 / \lambda$. The exponentiated Weibull distribution is a generalized form of Equation (3):

$$
P(\Delta t)=\alpha \frac{k}{\beta}\left(\frac{\Delta t-\theta}{\beta}\right)^{k-1}\left[1-e^{-\left(\frac{\Delta t-\theta}{\beta}\right)^{k}}\right]^{\alpha-1} e^{-\left(\frac{\Delta t-\theta}{\beta}\right)^{k}},
$$

where the additional parameters $\theta<0$ and $\alpha$ respectively account for simultaneous events and for temporal changes in the statistics. Specifically, if the events are clustered (i.e., $k<1), k \alpha \lessgtr 1$ indicates that the level of clustering varies over time, reaching a minimum (maximum) in a certain time period ( $\alpha=1$ returns the Weibull distribution of Equation (3)). As far as $\theta$ is concerned, it is worth noting that in those experiments where the detection of simultaneous events (if any) is prevented, for instance, by the finite temporal resolution of the sampling, $\theta$ should be larger than 0 . Finally, it can be readily shown that the exponentiated Weibull function exhibits a power-law tail, $P(\Delta t) \sim \Delta t^{-\mu}$, with $\mu=k \alpha-1$, for large waiting times.

The exponentiated Weibull distribution (Equation (4)) satisfactorily reproduces the observed WTD for ICW packets (bottom-right panel of Figure 1) with the following parameters $k=0.293 \pm 0.010, \beta=0.073 \pm 0.018, \theta=0.296 \pm 0.009, \alpha=11.4 \pm 1.3$ (inferred from the Levenberg-Marquardt least-squares fit, the resulting $\tilde{\chi}^{2}=2.7 \times 10^{-5}$ ). Some conclusions related to the statistical properties of the ICWs can be drawn by fitting the distribution of the ICW packet waiting times with Equation (4): (i) $k<1$, confirming the temporal clustering of the ICW packets, as previously deduced by comparing the observed ICW WTD with the theoretical distribution expected under Poisson hypothesis; (ii) as expected (since the identification of ICW packets is based on time series acquired by one single instrument at a finite cadence of $92 \mathrm{~ms}$ and, in turn, it is impossible to simultaneously measure two ICW trains) $\theta>0$, indicating that the probability of occurrence of two successive events is not zero for $\Delta t>\theta \simeq 0.3 \mathrm{~s}$, corresponding to about 3 magnetic data points; (iii) $k \alpha>1$, pointing to a not-uniform ICW clustering over the observational period and to the existence of a time interval (which can easily be assumed to encompass the last four hours, bottom-left panel of Figure 1) when ICW packets are more highly clustered; (iv) the exponentiated Weibull function fits the power-law tail of the ICW WTD observed at large waiting times well, where the exhibited power-law scaling $(\gamma=2.46 \pm 0.02$, see above) is 
equal, within the uncertainties, to that predicted by the asymptotic behavior of Equation (4) $(\mu=k \alpha-1=2.34 \pm 0.40)$.

\section{Discussion and Conclusions}

The results outlined in the previous section support the following scenario. The exponential distribution function of KAWs strongly suggests that they are governed by the Poisson hypothesis, and that they randomly occur as independent (i.e., uncorrelated) events. On the other hand, the power-law WTD observed for ICWs clearly indicates that Poisson statistics cannot account for the temporal distribution of ICW packets: nonstochastic, correlated clusters are indeed present in the ICW packet time sequence. Since the statistical properties of WTDs are related to the physical processes underlying the origin of the corresponding events, it appears evident that ICWs and KAWs are generated by two different mechanisms. Specifically, while stochastic processes seem to regulate the occurrence of KAWs, a persistent process, namely characterized by some memory, clearly drives the formation of ICWs, which result to be correlated to some degree.

The analysis of ICW and KAW WTDs results thus have great physical relevance in terms of understanding and characterizing the mechanisms driving the generation of different families of high-frequency Alfvén waves populating the solar wind at proton scales. Despite more refined statistical analyses, performed on the latest generation data provided by the novel solar missions Parker Solar Probe and Solar Orbiter, are required to definitevely solve the problem of the origin of ICWs and KAWs, some clues may be advanced even on the basis of the results of the present work, which represents a first step for further investigations. As a matter of fact, KAWs represent the high $k_{\perp}$ branch of the Alfvén solutions. As such, KAWs are the results of the turbulent evolution of Alfvén waves presumably formed by the stochastic jostling of the flux-tube foot-points driven by convection on the solar surface, and then propagating outwardly. The stochastic nature of the Alfvén waves at large scale is thus expected to extend to the higher-frequency fluctuations driven by turbulent cascades. Hence, this may explain why KAWs occur as random, uncorrelated events. On the other hand, as pointed out in Telloni et al. [20] on the basis of a statistical analysis, ICWs are dominated by proton cyclotron instability, which reacts to the anisotropic VDFs (due to energization of the protons in a direction perpendicular to the magnetic field) emitting $k_{\|}$left-handed polarized waves, say ICWs, at proton scales and, in turn, restoring VDFs towards thermal equilibrium. This process should have necessarily some memory, thus implying some correlation between the generated events. As a matter of fact, when an isotropic Maxwellian distribution of the VDFs is restored, the proton cyclotron instability is inhibited from driving the formation of ICWs. As a result, the time sequence of ICW packets should appear characterized by clusters of events (in periods of activity of the proton cyclotron instability) separated by longer-term correlated voids (when the proton VDF is in thermal equilibrium), as observed.

For the sake of completeness, it is of merit to advance another possible interpretation for the origin of the observed power-law-like WTD of ICWs, based on the hypothesis that ICW generation is governed by a time-dependent Poisson process. As a matter of fact, if ICWs occur as a sequence of independent events with a time-varying rate, $\lambda=\lambda(t)$, their WTD can be easily derived as, e.g., [25]

$$
P(\Delta t)=\frac{1}{\lambda_{0}} \int_{0}^{\infty} \lambda^{2} f(\lambda) e^{-\lambda \Delta t} d \lambda,
$$

where $f(\lambda)$ is the probability density function of the rate and $\lambda_{0}$ is the average rate, i.e.

$$
\lambda_{0}=\int_{0}^{\infty} \lambda f(\lambda) d \lambda .
$$

If the occurrence rate probability is constant, i.e., $f(\lambda)=\delta\left(\lambda_{0}\right)$, Equation (5) trivially reduces to the exponential waiting-time distribution function for a Poisson process, $P(\Delta t)=$ $\lambda_{0} e^{-\lambda_{0} \Delta t}$. On the other hand, if the distribution of the rate varies with time, it results that the 
asymptotic behavior of $P(\Delta t)$ for large waiting times $(\Delta t \rightarrow \infty)$ depends on the behavior of $\lambda^{2} f(\lambda)$ for small $\lambda$ values. Specifically, if $f(\lambda)$ follows a power-law function, $f(\lambda) \propto \lambda^{\alpha}$ for $\lambda \rightarrow 0$, Equation (5) predicts a power-law tail $P(\Delta t) \propto \Delta t^{-(3+\alpha)}$ for $\Delta t \rightarrow \infty$, when $\alpha>-3$.

It turns out that the observed power-law tail in the ICW WTD can arise from the realization of renewal Poisson processes with a variable rate. In this case, the only difference between the mechanisms underlying the origin of KAWs and ICWs resides in the rate of generation $\lambda$ of the two wave modes: while this is constant for KAWs, $\lambda=\bar{\lambda}$, yielding an exponential Poisson distribution of the KAW time sequence, the rate of the process varies with time $\lambda=\lambda(t)$ for ICWs, leading, as seen above, to mimic a power-law WTD distribution of ICWs at large waiting times.

In order to disentangle the likely role played by the modulation in time of the ICW rate in the WTD and to ascertain a power-law behavior of the ICW rate probability density, longer time series are required. Furthermore, the application of the Kolmogorov-Smirnov (K-S) test [26] is required as well to test for consistency of the local Poisson hypothesis with the observations. This analysis will definitively solve the unambiguity regarding the persistence or stochasticity underlying the origin of the ICWs in the solar wind. However, this is devoted to a future work.

Funding: D.T. was partially supported by the Italian Space Agency (ASI) under contract I/013/12/0.

Data Availability Statement: Wind data analyzed in this study are publicly available: they were downloaded from the NASA-CDAWeb (https:/ / cdaweb.gsfc.nasa.gov/index.html/).

Acknowledgments: D.T. wishes to thank R. Bruno for the useful discussions and comments.

Conflicts of Interest: The author declares no conflict of interest. The funders had no role in the design of the study; in the collection, analyses, or interpretation of data; in the writing of the manuscript, or in the decision to publish the results.

\section{References}

1. Neugebauer, M.; Snyder, C.W. Solar Plasma Experiment. Science 1962, 138, 1095-1097. [CrossRef] [PubMed]

2. Parker, E.N. Dynamics of the Interplanetary Gas and Magnetic Fields. Astrophys. J. 1958, 128, 664. [CrossRef]

3. Kolmogorov, A. The Local Structure of Turbulence in Incompressible Viscous Fluid for Very Large Reynolds' Numbers. Akademiia Nauk SSSR Doklady 1941, 30, 301-305.

4. Hansen, R.T.; Garcia, C.J.; Grognard, R.J.M.; Sheridan, K.V. A coronal disturbance observed simultaneously with a white-light corona-meter and the $80 \mathrm{MHz}$ Culgoora radioheliograph. Proc. Astron. Soc. Aust. 1971, 2, 57. [CrossRef]

5. D'Amicis, R.; Matteini, L.; Bruno, R. On the slow solar wind with high Alfvénicity: from composition and microphysics to spectral properties. Mon. Not. R. Astron. Soc. 2019, 483, 4665-4677. [CrossRef]

6. McComas, D.J.; Barraclough, B.L.; Funsten, H.O.; Gosling, J.T.; Santiago-Muñoz, E.; Skoug, R.M.; Goldstein, B.E.; Neugebauer, M.; Riley, P.; Balogh, A. Solar wind observations over Ulysses' first full polar orbit. J. Geophys. Res. 2000, 105, 10419-10434. [CrossRef]

7. Hundhausen, A.J. Coronal Expansion and Solar Wind; Springer: Berlin/Heidelberg, Germany, 1972.

8. Antonucci, E.; Harra, L.; Susino, R.; Telloni, D. Observations of the Solar Corona from Space. Space Sci. Rev. 2020, 216, 117. [CrossRef]

9. McComas, D.J.; Elliott, H.A.; Schwadron, N.A.; Gosling, J.T.; Skoug, R.M.; Goldstein, B.E. The three-dimensional solar wind around solar maximum. Geophys. Res. Lett. 2003, 30, 1517. [CrossRef]

10. Lamy, P.L.; Floyd, O.; Boclet, B.; Wojak, J.; Gilardy, H.; Barlyaeva, T. Coronal Mass Ejections over Solar Cycles 23 and 24. Space Sci. Rev. 2019, 215, 39. [CrossRef]

11. Reames, D.V. Particle acceleration at the Sun and in the heliosphere. Space Sci. Rev. 1999, 90, 413-491. [CrossRef]

12. Belcher, J.W.; Davis, Leverett, J. Large-amplitude Alfvén waves in the interplanetary medium, 2. J. Geophys. Res. 1971, 76, 3534. [CrossRef]

13. Verscharen, D.; Klein, K.G.; Maruca, B.A. The multi-scale nature of the solar wind. Living Rev. Solar Phys. 2019, 16, 5. [CrossRef] [PubMed]

14. Bruno, R.; Carbone, V. The Solar Wind as a Turbulence Laboratory. Living Rev. Solar Phys. 2013, 10, 2. [CrossRef]

15. He, J.; Marsch, E.; Tu, C.; Yao, S.; Tian, H. Possible Evidence of Alfvén-cyclotron Waves in the Angle Distribution of Magnetic Helicity of Solar Wind Turbulence. Astrophys. J. 2011, 731, 85. [CrossRef]

16. Podesta, J.J.; Gary, S.P. Magnetic Helicity Spectrum of Solar Wind Fluctuations as a Function of the Angle with Respect to the Local Mean Magnetic Field. Astrophys. J. 2011, 734, 15. [CrossRef]

17. Telloni, D.; Bruno, R.; Trenchi, L. Radial Evolution of Spectral Characteristics of Magnetic Field Fluctuations at Proton Scales. Astrophys. J. 2015, 805, 46. [CrossRef] 
18. Bruno, R.; Telloni, D. Spectral Analysis of Magnetic Fluctuations at Proton Scales from Fast to Slow Solar Wind. Astrophys. J. Lett. 2015, 811, L17. [CrossRef]

19. Telloni, D.; Bruno, R. Linking fluid and kinetic scales in solar wind turbulence. Mon. Not. R. Astron. Soc. 2016, 463, L79-L83. [CrossRef]

20. Telloni, D.; Carbone, F.; Bruno, R.; Zank, G.P.; Sorriso-Valvo, L.; Mancuso, S. Ion Cyclotron Waves in Field-aligned Solar Wind Turbulence. Astrophys. J. Lett. 2019, 885, L5. [CrossRef]

21. Lepping, R.P.; Acũna, M.H.; Burlaga, L.F.; Farrell, W.M.; Slavin, J.A.; Schatten, K.H.; Mariani, F.; Ness, N.F.; Neubauer, F.M.; Whang, Y.C.; et al. The Wind Magnetic Field Investigation. Space Sci. Rev. 1995, 71, 207-229. [CrossRef]

22. Jian, L.K.; Wei, H.Y.; Russell, C.T.; Luhmann, J.G.; Klecker, B.; Omidi, N.; Isenberg, P.A.; Goldstein, M.L.; Figueroa-Viñas, A.; Blanco-Cano, X. Electromagnetic Waves near the Proton Cyclotron Frequency: STEREO Observations. Astrophys. J. 2014, 786, 123. [CrossRef]

23. Wicks, R.T.; Alexander, R.L.; Stevens, M.; Wilson, L.B.I.; Moya, P.S.; Viñas, A.; Jian, L.K.; Roberts, D.A.; O’Modhrain, S.; Gilbert, J.A.; et al. A Proton-cyclotron Wave Storm Generated by Unstable Proton Distribution Functions in the Solar Wind. Astrophys. J. 2016, 819, 6. [CrossRef]

24. Weibull, W. A Statistical Distribution Function of Wide Applicability. J. Appl. Mech. 1951, 18, $293-297$.

25. Wheatland, M.S.; Litvinenko, Y.E. Understanding Solar Flare Waiting-Time Distributions. Sol. Phys. 2002, 211, 255-274. [CrossRef]

26. Bi, H.; Boerner, G.; Chu, Y. Correlations in the absorption lines of the quasar Q 0420-388. Astron. Astrophys. 1989, $218,19-23$. 